\title{
From lipids to lipid nanoparticles to mRNA vaccines
}

\author{
Lipid nanoparticles are essential to mRNA vaccines. The groundwork for lipid-based drug delivery systems was laid \\ more than 40 years ago in the lab of Pieter Cullis, Professor at the University of British Columbia. Nature Reviews \\ Materials talks to Pieter Cullis about the history and future of lipid nanoparticle-nucleic acid drugs.
}

\begin{abstract}
Q You have been working with lipids for a long time. What stimulated you to work on lipids and how did that lead to designing lipid-based systems that enable mRNA therapeutics, such as the COVID-19 mRNA vaccines?

Wow, that's a long story! After getting a PhD in physics, I decided that the problems outside physics looked a lot more interesting than problems in physics. So, I switched to biochemistry as a postdoc in Oxford, knowing nothing about lipids or membranes, but a bit about nuclear magnetic resonance (NMR). There I met Ben de Kruijff, a postdoc from Utrecht, and we used ${ }^{31} \mathrm{P}$ NMR to study the structural properties of lipids found in biological membranes. I became fascinated by lipid polymorphism, which involves the preference of some membrane lipids for non-bilayer structures, such as the hexagonal $\mathrm{H}_{\text {II }}$ phase. Our work led to the conclusion that such lipids play essential roles in membrane fusion processes that are basic to life, such as those that occur during fertilization and trafficking of intracellular vesicles.

After establishing my laboratory at the University of British Columbia (UBC) in the 1980s, I also became interested in the functional consequences of lipid asymmetry; the fact that the lipid composition on one side of a biological membrane is different to the lipid composition on the other side. I wondered whether we could generate lipid asymmetry in response to trans-bilayer ion gradients, such as $\mathrm{pH}$ gradients. We developed ways to make bilayer vesicles with a $\mathrm{pH}$ gradient and then generated lipid asymmetry by including an 'ionizable cationic lipid' that can exist in a neutral form or a positively charged (protonated) form, depending on the $\mathrm{pH}$. In vesicles exhibiting a $\mathrm{pH}$ gradient, this lipid preferentially distributes to the lipid monolayer experiencing the lower $\mathrm{pH}$, thus producing lipid asymmetry.
\end{abstract}

We got a bit distracted at this point, as we found out that we could also load anticancer drugs into bilayer vesicles exhibiting a $\mathrm{pH}$ gradient, leading me and four senior postdocs to co-found Inex Pharmaceuticals to develop liposomal anti-cancer drugs. However, in the mid-1990s, the CEO complained to me that he couldn't raise money putting old drugs into liposomes. We needed to be doing gene therapy! The only way we could efficiently encapsulate highly negatively charged nucleic acid-based drugs was to use cationic lipids. The cationic lipids then available were permanently positively charged and were highly toxic in vivo, so we had to find an alternative. Luckily, we tried the ionizable cationic lipid used for our lipid asymmetry studies and found that if we loaded the nucleic acid polymer at low $\mathrm{pH}$, where the ionizable lipid was positively charged, the nucleic acid payload was retained when the $\mathrm{pH}$ was raised to physiological $\mathrm{pH}$ values. And the systems were much less toxic.

Q So, your basic research on lipid asymmetry enabled you to find a way to encapsulate nucleic acids into lipid systems, but how did your work on lipid polymorphism help? In the late 1990s, it was well known that cationic lipids not only facilitate encapsulation of nucleic acids but can also enhance intracellular delivery in vitro. We became curious as to why that was. We found that cationic lipids combine with negatively charged lipids to form $\mathrm{H}_{\text {II }}$ phase structures, bringing us back to lipid polymorphism. This led to the hypothesis that cationic lipids facilitate intracellular delivery of RNA and DNA by combining with the negatively charged lipids found in biological membranes to form membrane-disruptive non-bilayer structures, enabling nucleic acid polymers to penetrate into cells.

This hypothesis had direct implications for the design of ionizable lipids. Lipid-based

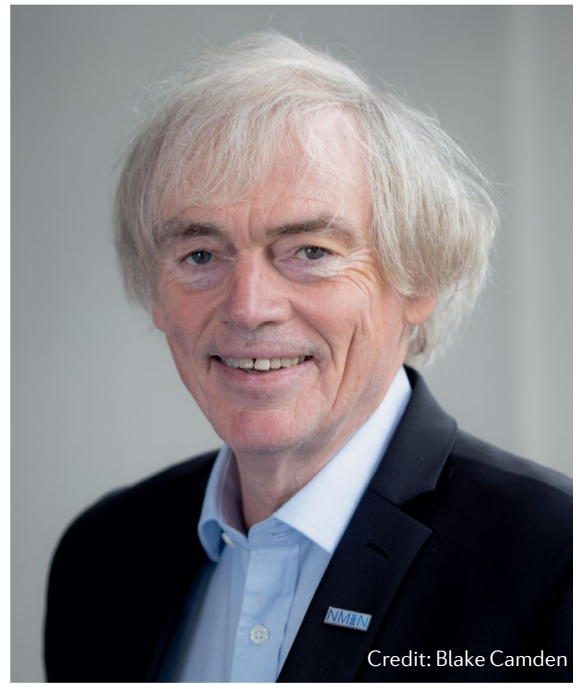

systems accumulate in cells through endocytosis and our objective was to destabilize the endosomal membrane to enable cytoplasmic delivery of the nucleic acid payload. The $\mathrm{pH}$ in the endosome goes down as the endosome matures. Thus, the potency of ionizable lipids should depend on the $\mathrm{pH}$ at which they become protonated as well as their ability to induce a non-bilayer structure once they are positively charged. This gave us a rational design approach to optimize ionizable lipids that has been most productive.

Q You have then co-founded start-up companies, which have significantly contributed to developing the lipid nanoparticle-mRNA formulations used for COVID-19 vaccines. Could you tell us about your experience in drug development outside academia? I realized early on that developing a drug required vastly greater resources than could be available in the academic system. Also, it is next to impossible to keep a world-leading scientific team together in academia over the long term. So I worked hard to start companies 


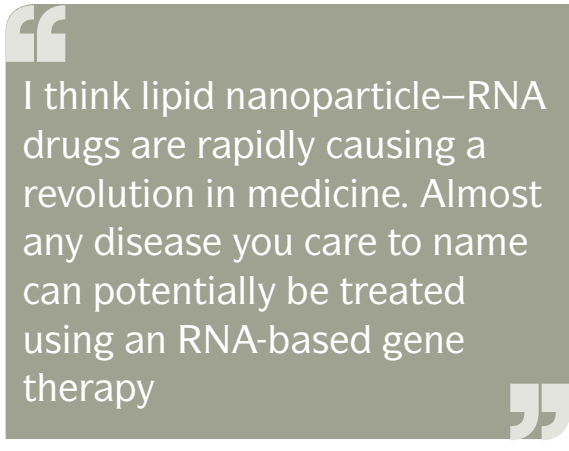

to keep a core group of truly excellent scientists together from the outset. It is a source of enormous satisfaction to me that, in one way or another, I am still working with Mick Hope, Tom Madden, Marcel Bally and Lawrence Mayer, who were senior members of my UBC group more than 40 years ago. If we had not established and maintained this core group, we would not have achieved much.

These companies played pivotal roles in the evolution of the COVID-19 mRNA vaccines. Research at Inex triggered our initial foray into developing lipid-based delivery systems for nucleic acid-based drugs. This was followed by work at Protiva Biotherapeutics and subsequently Acuitas Therapeutics, in collaboration with Alnylam Pharmaceuticals and my UBC laboratory, to develop the optimized lipid nanoparticle system used for the Onpattro product (a lipid nanoparticle-based siRNA drug for the treatment of polyneuropathies induced by hereditary transthyretin amyloidosis). I should say the collaboration with Alnylam from 2005 to 2012 was a game changer. Together, we synthesized more than 300 species of ionizable lipid and improved the potency of lipid nanoparticlesiRNA systems for silencing genes in the liver by more than three orders of magnitude.

After Onpattro went into clinical trials in 2012, Acuitas went on to develop lipid nanoparticle systems containing mRNA to express proteins in the liver. We thought that if we could deliver siRNA for gene silencing, perhaps we could deliver mRNA for gene expression. Remarkably, this worked, and Acuitas has developed increasingly potent lipid nanoparticle-mRNA systems for gene expression in the liver. Around 2014, we had the enormous good fortune to be contacted by Drew Weissman of the University of Pennsylvania, who wanted to try our systems as vaccines. These worked brilliantly, paving the way for mRNA vaccines.

Q What is the advantage of using lipid nanoparticles for nucleic acid delivery, compared to viral delivery systems and other nanoparticles, such as synthetic polymers, peptide-based nanomaterials or extracellular vesicles?
Well, I am obviously biased, but one of the delights of working with lipids is that you can take advantage of what nature provides. Lipids have amazing material properties, are highly flexible, are biodegradable and allow you to use biological processes to your advantage. An example is the way in which lipid nanoparticle-RNA systems gain access to hepatocytes in the liver, by adsorbing ApoE following intravenous injection, which triggers uptake into hepatocytes via low-density lipoprotein (LDL) and other receptors.

The advantages compared with other delivery systems are profound. Viral vectors suffer from limited carrying capacity, manufacturing problems and immune response concerns. Other synthetic systems are hampered by poor loading efficiencies, inhomogeneity and immune response issues. Extracellular vesicles are difficult to manufacture and load, and they are highly complex structures that make it almost impossible to achieve batch-to-batch reproducibility.

Q We now know a lot about lipid nanoparticle interactions in the body; what are the remaining fundamental problems and challenges that need to be addressed? A great many fundamental problems and challenges remain. A detailed understanding of the mechanism whereby ionizable lipids engender endosomal escape is important. The protein corona that adsorbs to lipid nanoparticles in vivo and its influence on particle performance remain poorly characterized. How do we target tissues other than hepatocytes following intravenous administration? How do we turn gene expression on or off, so that we are transfecting only tissues in certain regions? How do we access important target tissues, such as the brain? The list goes on and on. I must say though that I am optimistic that lipid-based systems will meet these demands. Modulation of the outer surface of lipid nanoparticles will engender different tissue distributions. Co-encapsulation of agents sensitive to external radiation could allow site-specific gene therapies. Increasingly potent ionizable lipids and optimization of other lipid nanoparticle components will enable hard-to-transfect tissues to be manipulated.

Q Nanomedicine has suffered some trust issues in the past, in particular, cancer nanomedicines, partly owing to very slow clinical translation. Do you think the success of the COVID-19 mRNA vaccines will speed up the clinical translation of other nanomedicines, for example, against influenza, cancer or HIV?

I think lipid nanoparticle-RNA drugs are rapidly causing a revolution in medicine. Almost any disease you care to name can potentially be treated using an RNA-based gene therapy. Further, these drugs can be developed with amazing speed. Once you know the protein you wish to silence or express, the requisite siRNA or mRNA can be synthesized in a month or two, and can be packaged in a lipid nanoparticle in a day or two to provide a targeted drug. The progression of the COVID-19 mRNA vaccines, from concept to a drug in clinical trials, within 3 months of sequencing the viral genome, exemplifies these possibilities. It is highly likely that we will see lipid nanoparticle-mRNA vaccines for influenza, HIV, Zika, COVID-19 variants and other infectious diseases very soon.

But this is just the tip of the iceberg. The ability to use the liver as a bioreactor to produce any protein required following an intravenous injection has enormous potential. The range of applications includes monoclonal antibodies to treat everything from cancer to infectious diseases. Proteins to treat rare diseases, such as lysosomal storage disorders, glycogen storage disorders and haemophilia, are obvious possibilities. As soon as we can access the bone marrow, therapies for sickle cell anaemia and various leukaemias will come into focus. Similar possibilities exist for the lung, heart, eye and brain. It is an amazing time for medicine.

Q. It must have been very exciting to see how well the COVID-19 mRNA vaccines work and very gratifying to have contributed to that amazing success. What next? It was unbelievable. When BioNTech/Pfizer announced that the vaccine was $95 \%$ effective for preventing COVID-19, I couldn't believe it. Even after a few glasses of scotch, I still couldn't believe it. You have to remember that up until a year or so ago, liposomes and lipid nanoparticles were relatively unknown, despite decades of effort by me and many others to prove that they could be valuable delivery systems. To have this proven in such a dramatic fashion in response to a global emergency, well, it's beyond belief. Lipid nanoparticles are going into billions of arms. All I can say is that it makes me so happy that a journey I and four postdocs in my lab embarked on 40 years ago has had such an impact.

With regard to what's next, if there is one thing life has taught me, it's that there is no time like the present to try to achieve impossible dreams. So, I have started a company to try to treat ageing. A bit late for me perhaps, but you never know.

Interviewed by Christine Horejs https://oi.org/10.1038/s41578-021-00379-9 